\title{
Dal testo mathema al testo "evento" di verità
}

\section{Rocco Ronchi, Università dell'Aquila}

\begin{abstract}
This paper addresses the modern crisis of the philosophical project that conceived of literature as mathesis universalis. In the digital universe, literature maintains its relationship with truth, which is no longer considered as transcending the process of knowing, but as given in unity with it. The new statute of the text is captured well by Carlo Sini's idea of "foglio-mondo" ("world-page"). Sini suggests that the desacralized text is not situated "outside" of truth; it does not become, as happens in many postmodern thinkers, play, deceit, and gratuitous narration. Rather, "foglio-mondo" is the text freed from the tutelage of transcendental truth to become an "event" of truth.
\end{abstract}

Questo intervento affronta il problema della crisi moderna del progetto filosofico della letteratura come mathesis universalis. Nell'universo digitale la letteratura mantiene il suo rapporto con la verità che non viene più considerata trascendente il processo della conoscenza, ma data in unità ad esso. Il nuovo statuto del testo è bene indicato dall'espressione di Carlo Sini "foglio-mondo." Il testo desacralizzato non viene a situarsi per Sini "fuori" dalla verità, non diventa, come in tanti pensatori postmoderni, gioco, menzogna, narrazione gratuita. Il "foglio-mondo" è piuttosto il testo che si è liberato dalla tutela della verità trascendente per diventare "evento" di verità.

Introducendo il bel volume $A l$ di là del testo. Critica letteraria e studio della cultura, Francesco Fiorentino ha richiamato l'attenzione del lettore sul seguente passo di Roland Barthes:

Si può definire "letteratura" - scrive il critico francese - un corpus di testi sacralizzati ma anche classificati da un metalinguaggio (la "storia della letteratura”), cioè un corpus di testi del passato che vanno dal XVI al XX secolo [...] Questa letteratura fino al XX secolo è una mathesis: un campo completo del sapere. Tutti i saperi del mondo in un momento determinato vengono messi in scena attraverso testi diversissimi [...] L’importante non è elaborare, diffondere un sapere sulla letteratura (nelle "storie della letteratura”), ma manifestare la letteratura come mediatrice di sapere. (7)

Il passo si trova in una intervista a Roland Barthes del 1975, ripresa poi in La grain de la voix (232-233.) Meriterebbe un'attenta esegesi, ma io mi limiterò a interrogarmi sulla proposta forte, di natura epistemologica, in esso contenuta. La letteratura, scrive Barthes, è innanzitutto una mathesis. Ma cosa significa considerarla tale? Che cosa implica una 
considerazione della letteratura come mediatrice del sapere? Mathesis, da mathein, è quanto può essere fatto oggetto di insegnamento. C'è mathesis dove c'è mathema, vale a dire un sapere trasmissibile in modo univoco senza perdita di informazione nel corso della sua comunicazione. Il modello di tale sapere è offerto dalla matematica, vale a dire da quel sapere che per definizione è il più lontano dalla retorica, in quanto prescinde per definizione da ogni riferimento al contesto enunciativo. Non è un sapere storicamente "situato". La letteratura come mathesis aspira allora ad uno statuto che sembra in contraddizione con la sua natura soggettiva e storica. Tra le condizioni, infatti, che per gli antichi rendevano un sapere insegnabile ve ne era infatti una assolutamente imprescindibile. Era l'identità dell'oggetto inteso come invarianza. Solo in quanto oggetto ideale di un sapere assoluto, trans-storico, solo in quanto astratto da ciò che la fenomenologia chiama il "mondo della vita," un enunciato poteva entrare a far parte di quella particolare tradizione che è la tradizione della verità. Una tradizione che diciamo particolare per la sua peculiare universalità. A differenza, infatti, di tutti i saperi tradizionali, narrativi, territoriali - dunque a differenza anche di quanto saremmo portati a classificare sotto la rubrica letteratura - tale enunciato è votato, come la proposizione matematica, all'universale, a ciò che è sempre e che mai non tramonta.

L'affermazione di Barthes è, quindi, paradossale e controintuitiva. Invita ad una matematizzazione di quanto solitamente si ritiene essere l'ambito del soggettivo, del vissuto, dello storicamente determinato. Per essere intellegibile richiede perciò che si dia all'espressione "corpus di testi letterari" un significato più ampio, il quale necessariamente trascenderà i confini storici troppo angusti assegnatigli dal critico francese ("dal XVI al XX secolo").

Letteratura è parola di origine latina che rinvia alle "lettere", ai grammata. In senso generalissimo, ma non vago, letteratura rinvia alla dimensione della "testualità", vale a dire all'obiettivazione della memoria resa possibile dai grammata della scrittura fonetica alfabetica. Così intesa la sua assimilazione ad una mathesis diventa non solo comprensibile, ma pienamente giustificata. L'alfabeto fenicio ristrutturato dai greci - o, come impropriamente si dice, "vocalizzato" dai greci (impropriamente perché l'invenzione specifica dei greci fu piuttosto la consonante (231-263) - è infatti un miracoloso algoritmo che rende possibile la produzione del mathema (Ronchi 205-208). L'idealità, l'universale, viene per così dire liberata dalle sue aderenze sensibili e proiettata nell'iperuranio platonico, solo oggetto di insegnamento possibile. Solo dell'idea vi è infatti scienza e insegnamento. Una sterminata letteratura scientifica ha discusso, nella seconda metà del secolo scorso, questo epocale passaggio ed è inutile indugiare ancora su di esso. Ricordo solo, per il suo carattere inaugurale, il saggio che segna l'esordio filosofico di Jacques Derrida ("Intruduction"). Mi soffermo piuttosto sulla natura del sapere inaugurato dalla ristrutturazione alfabetica dell'esperienza. Che tipo di sapere è, infatti, il sapere reso possibile dalla trascrizione alfabetica dell'esperienza? Ł̀, appunto, una mathesis universalis, un sapere assoluto. O meglio, un sapere dell'assoluto, nel duplice senso del genitivo: oggettivo e soggettivo. È la filosofia intesa come scienza speculativa. È la filosofia come scienza della verità. Grazie all'algoritmo alfabetico, è la Verità, proprio lei, quella con la 
maiuscola a capolettera, a prendere la parola e a presentarsi nel tempo degli uomini. “Tradizione della verità" significa che il vero non cessa di dirsi nel tempo, attraverso figure che sono tutte figure della verità e che lo sono proprio in quanto, nello stesso tempo, sono anche figure dell'errore, se isolatamente prese e astratte dal tutto.

La Fenomenologia dello spirito di Hegel drammatizza il presentarsi di siffatta verità assoluta, un presentarsi da intendersi nel senso riflessivo del termine, giacché l'assoluto non ha altro che se stesso come interlocutore: si comunica (impropriamente e difettivamente) agli uomini per comunicarsi (propriamente e perfettamente) a se stesso, per prendere coscienza di sé come assoluto fuori dal quale non vi può essere ovviamente nulla. La "letteratura”, così intesa, è effettivamente "una mathesis: un campo completo del sapere”. "Testi diversissimi”, tra loro in conflitto, hanno messo effettivamente "in scena”, come scrive Barthes, questo sapere assoluto. In quanto veicoli della manifestazione di questo sapere essi sono però tutti filosofici, indipendentemente dalla categoria culturale alla quale superficialmente appartengono (poesia, romanzi, trattati...). Partecipano, cioè, della stessa tradizione della verità proprio per la loro natura materiale di "testi”, di obiettivazioni del sapere rese possibili dall'algoritmo alfabetico. Ed è solo in quanto traducibili o riducibili alla dimensione della testualità e, in ultima analisi, della scrittura, che altre manifestazioni del cosiddetto spirito umano potranno aspirare ad entrare a far parte della scena del sapere: la pittura, ad esempio, dovrà farsi con Vasari e la rivoluzione rinascimentale, arte liberale, dovrà diventare "come poesia”, per entrare a far parte dell'enciclopedia universale del sapere filosofico.

È possibile allora indicare anche un luogo nel quale si è fatta sensibile la crisi moderna del progetto filosofico della letteratura come mathesis universalis. Sono d'accordo con Fiorentino nel rintracciarlo nel "romanzo più enciclopedico che sia mai stato scritto" (secondo la definizione che ne dà Italo Calvino): Bouvard e Pécuchet di Flaubert. Che altro è, infatti, Bouvard et Pécuchet se non la fenomenologia dello spirito di Hegel messa in epoché da un mal vissé dans l'univers du discours (così Sartre definisce il giovane Flaubert in L'Idiot de la famille)? (Sartre 26). Anche lì, nelle campagne normanne, abbiamo un assoluto che si manifesta in figure del sapere e in figure della coscienza tra loro dialetticamente concatenate, figure che, come nella hegeliana scienza dell'esperienza della coscienza, trapassano l'una nell'altra tramite una "catastrofe." Bouvard e Pécuchet passano da una figura del sapere a un'altra, nell'inesausta ricerca di una completezza del sapere sempre mancata. Il comicissimo ed incompiuto processo di autoformazione dei due buffi amici è effettivamente una hegeliana "via del dubbio e della disperazione”, ma ora l'assoluto è diventato l'assoluto della bêtise, della stupidità eretta a sistema. Qualcosa deve essere successo se la letteratura ha cessato di essere una mathesis, se ha smesso di essere "mediatrice del sapere."

Che cosa è successo? In prima battuta possiamo dire che la letteratura ha perso la sua autorità e che tale crisi si è comunicata epidemicamente a tutti quei luoghi, materiali e immateriali, in cui la sua autorità si segnalava: dalle scuole alle università, dalle biblioteche al libro, inteso anche nella sua forma sensibile di manufatto. Nessuno oggi entra più in questi luoghi con la sensazione di varcare una soglia metafisica, nessuno sfoglia un libro 
attendendosi da esso una sorta di lasciapassare per l'eternità. I libri, lo dicono tutti, si presentano oggi come merci, gadget, informazione, aggiornamento, occasioni di divertimento o strumenti utili alla vita e al lavoro. Il che non significa, come subito vedremo, che la letteratura abbia definitivamente interrotto il suo rapporto con la verità e con l'assoluto. Piuttosto questo rapporto si è trasformato in una direzione inedita che dovremo sondare. Percepire la letteratura come una mathesis significava percepirne una particolare sacralità quali che ne fossero i contenuti contingenti. Ogni testo appariva scritto all'ombra e, per così dire, sotto la protezione di una verità che lo precedeva e lo fondava, una verità che non era stata scritta da nessuno in particolare, che non era "umana" nella sua origine, ma che si era scritta da se stessa, una volta per tutte e per sempre, all'inizio o alla fine dei tempi. Ogni testo era sacro e ispirava un naturale senso di devozione e di rispetto un rispetto "a priori" - nel lettore, ben testimoniato dalla naturale deferenza rispetto alla forma libro nutrita da chi, come me, si è formato intellettualmente in un'epoca in cui il libro a stampa era ancora sinonimo di sapere e le biblioteche, le aule universitarie o le sedi delle conferenze erano luoghi non ordinari accedendo ai quali bisognava, come nelle case giapponesi, togliersi in qualche modo le scarpe.

Con un'espressione desunta dall'opera di Giovanni Gentile potremmo parlare per la letteratura come mathesis universalis di "metodo della trascendenza" (14). Gentile si riferiva al problema della conoscenza così come esso si pone nell'orizzonte metafisico. Se la conoscenza, egli scrive, è adeguazione dell'intelletto al vero, il vero non può che essere supposto altrimenti che come interamente già dato, compiuto in se stesso e accessibile da sempre allo sguardo di dio, al suo intuitus. In tale orizzonte, conoscere si risolve in un'approssimazione che non crea nulla, ma semplicemente porta allo scoperto un territorio che già sussiste "in sé" (e "per" lo sguardo di dio). Lo stesso si deve allora dire per la scrittura contingente di un testo quando la letteratura sia intesa come mathesis universalis, come campo completo del sapere. Nessun nuovo testo empiricamente dato può aggiungere una sola riga ad una verità che si deve supporre già interamente compiuta "da qualche parte,” come ha scritto una volta Henri Bergson (767; Ronchi, Bergson 161-175). Ogni scrittore partecipa infatti di una tradizione che è tradizione della verità, nel doppio senso oggettivo e soggettivo del termine. Ogni testo, in quanto testo, dice, come può, il vero o almeno è, già da sempre, in relazione con una verità trascendente. Lo è anche quando manca la verità perché questa è comunque l'unità di misura della sua menzogna.

La teologia implicita nella scrittura trova una sua precisa espressione nel passo del maestro vedanta Ramesh S. Balsekar che Domenico Fiormonte ha posto come esergo al saggio contenuto in questa raccolta:

[Nisargadatta] Maharaj said quite often that books get written; they are never written by authors. Only a little thought is necessary to see the truth of what he meant. He was NOT referring only to books on spiritual matters; he was referring to all books. In the overall functioning of the manifested universe, whatever was necessary as written or spoken words appeared spontaneously (...). No credit or blame could attach to any individual writer for the simple reason that the 
individual is a mere illusion and has not autonomous existence. (Ramesh S. Balsekar, Experience of Immortality)

Non c’è nessun irrazionalismo mistico dietro a questa formulazione. Balsekar traduce nell'orizzonte della sua cultura la percezione inevitabile che dell'autorità del libro deve avere ogni umanità che si affida al testo come mediatore del sapere assoluto. Ogni libro empirico rimanda al Libro, alla mathesis universalis che precede, fondandola, la scrittura contingente di un testo. La sua origine reale è nella Verità e solo derivatamente nell'autore che della Verità è, come scriveva Hegel, soltanto un funzionario, più o meno solerte, ma certamente sempre inadeguato al compito (una inadeguatezza che, tuttavia, misura il suo rapporto alla verità). Tale primato del Libro sui libri, tale primato o tale trascendenza del sapere assoluto, costantemente in atto, sul sapere determinato che ogni libro incarna, era quanto l'affermazione aristotelica del primato dell'intelletto agente sull'intelletto passivo conteneva in nuce. Chi conosce, chi scrive, lo può infatti fare perché si situa in una luce (sempre in atto) che lo investe e che lo precede rendendolo capace di conoscere qualcosa: conosce perché ha già conosciuto, conosce perché sostanzialmente "ricorda." Conosce perché il vero si manifesta a se stesso attraverso il suo atto singolare e finito di conoscenza. Scrive perché legge nel gran libro del sapere. Così è pensato il testo dalla metafisica e così è stato vissuto per millenni: teologicamente, come figura di una verità già data a priori.

Quello che è successo, ad un certo punto, forse proprio nella campagna normanna dove Bouvard e Pécuchet volevano abbeverarsi alla fonte del sapere assoluto, è che la letteratura, intesa in questo senso larghissimo, ha cessato di essere mathesis universalis. Il rapporto alla verità non è stato perduto ma ha cambiato radicalmente di segno. Ancora Gentile può fornirci una traccia di questo mutamento epocale che segna l'avvento del moderno e della sua peculiare "crisi." Gentile, infatti, al "metodo della trascendenza” opponeva il "metodo dell'immanenza” (246-247). Ad esso affidava la possibilità di una rinascita dell'idealismo. Con metodo dell'immanenza intendeva un altro rapporto alla verità, questa volta non più considerata trascendente il processo della conoscenza, ma data in unità ad esso: la verità non sarebbe fuori dal pensiero, al di là di esso, ma coinciderebbe con l'atto stesso del pensiero, con il pensiero che sta pensando in atto. Una verità immanente, quindi, che non cessa di crearsi e di decrearsi nel pensiero che qui e ora sta pensando.

Trasferito al nostro discorso, "metodo dell'immanenza” significa allora che non c’è più una Verità presupposta alla scrittura, non c'è più un Libro di cui i libri sarebbero approssimazioni inadeguate. Scrivere non è leggere nel grande Libro. La scrittura si emancipa dalla tutela della Verità a priori, diventa, per dirla con Kant, maggiorenne (142). E diventare maggiorenni significa respirare a pieni polmoni una libertà che può essere anche angosciante, perché non si hanno più garanzie trascendenti. L’atto di scrivere (e, con esso, l'atto di leggere) resta sospeso alla sua possibile e definitiva insensatezza. La non verità definitiva di un libro diventa un possibilità concreta. I libri gadget dei supermercati sono infatti semplicemente falsi. Il tempo che si dedica alla loro lettura è tempo semplicemente perso. Cosa diviene, allora, il testo che si vuole ancora testo, che si vuole ancora "sapere”, in questo nuovo orizzonte? Esso certamente perde la sua autorità sacrale. Di tale perdita d'aureola facciamo quotidiana esperienza e ce ne lamentiamo con toni nostalgici e 
passatisti. Tuttavia, il testo non si situa necessariamente fuori dalla verità, ma stabilisce con la verità un altro rapporto, della cui straordinaria fecondità non siamo ancora consapevoli, nonostante il grande blaterare intorno alle nuove forme di scrittura/lettura rese possibili dalle tecnologie digitali.

Carlo Sini è stato tra i pochissimi filosofi che si è posto il problema. Il suo pensiero è stato però sostanzialmente ignorato proprio da coloro che, più di altri, avrebbero dovuto interessarsene. Mi riferisco, appunto, a coloro che professionalmente si preoccupano di monitorare le trasformazioni indotte dalle nuove tecnologie. Sini, per indicare il nuovo statuto del testo, ha usato l'espressione "foglio-mondo" (Sini, Teoria e pratica del fogliomondo). Non posso naturalmente nemmeno provare a riassumere la complessa elaborazione teorica che sta alle spalle di questa nozione. Basti qui dire che il testo desacralizzato non viene a situarsi per lui "fuori" dalla verità, non diventa, come in tanti pensatori postmoderni, gioco, menzogna, narrazione gratuita. Il "foglio-mondo" è piuttosto il testo che si è liberato dalla tutela della verità trascendente per diventare "evento" di verità o, come più recentemente Sini ha scritto, “occasione” (Sini, Figure dell'enciclopedia filosofica 8087). La sua compromissione con la verità è forse ancora più forte di quella del testo tradizionale, del testo che era al servizio della verità a priori, perché la Verità, proprio quella con la maiuscola a capolettera, ora ha luogo integralmente nei confini del testo, accade in inscindibile unità con esso, ne condivide il destino e ne soffre le stesse limitazioni.

Provo a spiegarmi utilizzando un'espressione che non è di Sini, ma che, a mio giudizio, ben si presta a illustrare la natura del "foglio-mondo". La desumo da autori a me cari: Henri Bergson e Gilles Deleuze. Il testo che si genera con la fine della mathesis universalis ha lo statuto logico della "molteplicità virtuale," dove "virtuale" non ha nessun rapporto con le banalità irritanti oggi in circolazione. Si sarebbe potuto utilizzare anche l'espressione deleuziana "rizoma" se anch'essa non fosse pregiudicata dall'uso sconsiderato che filosofi dilettanti hanno fatto di essa. Molteplicità virtuale è, per Bergson, la durata creatrice. Molteplicità virtuale è unità e molteplicità al tempo stesso. È unità che si fa attraverso i molti non essendo nessuno dei molti e non avendo altra consistenza ontologica, in quanto unità, che nei molti in cui non è. Molteplicità virtuale è unità melodica, è una memoria revisionista, è l'atto del divenire che non coincide con il divenuto (con lo spazio percorso, con il testo effettivamente dato) non avendo altra dimensione di manifestatività che quel divenuto dal quale differisce per natura. In quanto foglio-mondo il testo è siffatta molteplicità. Il che significa che la sua unità non è affatto compromessa. Essa è più che mai riconoscibile. La sua identità, giustamente cara ai filologi, è più che mai preservata. Tuttavia non si tratta dell'identità del mathema, dell'invariante dell'oggetto ideale, perché la sua unità, lungi dall'essere presupposta (e data all' intuitus di dio), invece di essere già fatta, si fa attraverso i molti in una relazione di implicazione e differenza. L'identità del testo è ora nella variazione assunta come primum, rispetto alla quale ciò che varia, il tema, è già da intendersi come effetto dell'interpretazione (Rossella Fabbrichesi Leo e Federico Leoni (153163). Il testo, ogni testo, è ora quanto Bergson, con un'espressione difficilmente traducibile in italiano, chiama un mouvant. Forse la sola traduzione possibile del mouvant bergsoniano 
è quella gentiliana di atto in atto, di atto colto nel suo aver luogo, mai interamente dato. Più semplicemente si potrebbe dire, con Sini, che il testo diventa "evento" di verità. Lascio agli specialisti del settore il compito di stabilire se e come il foglio-mondo si avvicini dal punto di vista logico alla forma dell'ipertesto. Ciò che mi interessa segnalare in conclusione sono piuttosto alcuni corollari che discendono da questa nuova assiomatica del testo.

Primo corollario. Il testo come evento di verità (foglio-mondo) implica una nuova etologia della lettura. E stato Ivan Illich a sottolineare l'importanza dell'atto di lettura e delle sue trasformazioni per comprendere la storia culturale dell'Occidente. Ora, il testo emancipatosi dal suo rapporto con la mathesis universalis implica effettivamente una nuova postura del lettore. La postura standard, quella prevista dal testo alfabetico, è quella descritta magistralmente da Jesper Svenbro nei suoi saggi: è una postura che suscitava i lazzi osceni del greco ben educato che vi scorgeva una rassomiglianza con quella sodomitica (189-199). Il lettore è infatti passivo, viene dopo il testo e gli dona quella voce che il testo, sigillo di un'assenza definitiva, non può avere. Ma se il testo è compreso come molteplicità virtuale, come foglio-mondo, come mouvant, l'atto di lettura viene ora inscritto nel testo come parte del testo stesso, come suo momento costitutivo. La voce non si aggiunge al testo, come un supplemento d'anima dato ad un corpo morto, ma il testo parla (e vive) nuovamente ed in modo inedito con la voce del lettore. La lettura è esecuzione, certamente. Ma l'esecuzione è interpretazione e l'interpretazione è la vita vivente del testo stesso, qui e ora. Il testo è così ricollocato dall'atto di lettura all'interno di quella contesto enunciativo dal quale l'algoritmo alfabetico l'aveva astratto, costituendolo, appunto, come puro mathema. Probabilmente questa inscrizione del lettore è quanto offre al testo tradizionale una nuova dimensione verticale, di natura schiettamente temporale. Si potrebbe, a questo proposito, azzardare una definizione non banale del cosiddetto ipertesto. Esso non è infatti in alcun modo riducibile ad un testo a rete sul modello della enciclopedia di cui ha parlato, ormai molti anni fa, Umberto Eco (55-140). L'ipertesto è piuttosto, quale che sia il supporto materiale, un testo che inscrive nel suo corpus l'atto soggettivo e singolare di lettura, nel senso più ampio che può acquisire questa espressione: lettura come esecuzione e lettura come interpretazione, lettura come esegesi e lettura come critica... Un testo-mouvant, insomma, un atto in atto e non un fatto compiuto. Il teso diviene, bergsonianamente, una durata creatrice di imprevedibili novità.

Secondo corollario. Il testo come evento di verità implica un nuovo concetto di responsabilità scientifica. Per che cosa si è responsabili, ad esempio, come filologi, vale a dire come amanti dei discorsi che sono stati fatti e che si sono depositati nella memoria collettiva sotto forma di testi? Per la verità del testo, certamente. Di che altro si deve infatti rispondere? Ma tale verità va ora declinata anche nel senso dell'immanenza e non più in quello della sola trascendenza. La verità per la quale si deve rispondere non è più o, almeno, non è soltanto quella dell'origine. Il tempo trascorso non ha solo il senso del guasto arrecato all'originale, della degradazione arrecata all'archetipo. Il testo come evento di verità inscrive positivamente il tempo dentro di sé. Ciò che dal punto di vista metafisico poteva apparire soltanto come un attentato all'integrità del testo, al quale bisognava porre in qualche modo rimedio, dal nuovo punto di vista è parte integrante della sua verità in atto, della sua verità 
in corso d'opera. Il filologo, amante dei discorsi, diviene così responsabile anche per la verità a venire del testo. La sua pratica assume il senso di un "turno conversazionale". Così l'etnometodologia intende l'enunciato comunicativo. È un rispondere ed un corrispondere ad una verità che è in gioco ${ }^{1}$. Il testo, in quanto evento di verità, non è insomma un significato, un'idea, un mathema, dato una volta per tutte (ma a chi, poi?), ma un senso, un senso sempre da farsi che circola in quella conversazione infinita che è la tradizione della verità (ma una verità immanente).

1 In questa direzione si muove la cosiddetta etnofilologia. Purtroppo gli strumenti speculativi di cui dispone sono ancora inadeguati alla bisogna (Benozzo).

\section{Works Cited}

Barthes, Roland. La grain de la voix. Entretiens 1962-1980, Seuil, Paris 1980; trad. it. Torino: Einaudi, 1981. Print.

Benozzo, Francesco. Dalla filologia tradizionale all'etnofilologia tradizionante." Canoni liquidi. Ed.

Domenico Fiormonte. Roma: Scripta Web, 2011. 27-42. Print.

Bergson, Henri. Oeuvres. Paris: Puf, 1959. Print.

Deleuze, Gilles e Guattari, Félix. "Rizoma” (1976). Millepani. Capitalismo e schizofrenia. Roma: Istituto dela Encicolpedia Italiana, 1987. 3-36. Print.

Derrida, Jacques. "Introduction." Husserl, Edmund. L’Origine de la géométrie. Paris: Puf, 1962. Print.

Eco, Umberto. Semiotica e filosofia del linguaggio. Torino: Einaudi, 1984. Print.

Kant, Immanuel. "Risposta alla domanda: Che cos'è illuminismo.” Scritti politici, Torino: UTET, 1971. Print.

Fabbrichesi, Rossella Leo e Leoni, Federico. Continuità e variazione. Leibniz, Goethe, Peirce, Wittgenstein con un'incursione kantiana. Milano: Mimesis, 2005. Print.

Fiorentino, Francesco. "Infinite reti: la letteratura nell'ipertesto della cultura." Al di là del testo. Critica letteraria e studio della cultura. Ed. Francesco Fiorentino. Macerata: Quodlibet, 2011. 9-62. Print.

Gelb, Ignace J. A study of writing. Chicago: Chicago University Press, 1952; trad. It. Teoria generale della scrittura. Fondamenti della grammatologia. Milano: EGEA, 1993. Print.

Gentile, Giovanni. “Il metodo dell'immanenza”(1912). La riforma della dialettica hegeliana e altri scritti. Messina: Principato, 1923. Print.

Illich, Ivan. Nella vigna del testo. Per un'etologia della lettura. Milano: Raffaello Cortina, 1994. Print. 
Ronchi, Rocco. Bergson. Una sintesi. Milano: Christian Marinotti Edizioni, 2011. Print.

---. Filosofia della comunicazione. Il mondo come resto e come teogonia. Torino: Bollati Boringhieri, 2008. Print.

Sartre, Jean-Paul. L'Idiot de la famille. Gustave Flaubert de 1821 à 1857. Vol. I. Paris: Gallimard, 1971. Print.

Sini, Carlo. Figure dell'enciclopedia filosofica. "Transito-Verità”. Opere. Vol. 5. Milano: Jaca Book, 2012. Print.

---. Teoria e pratica del foglio-mondo. La scrittura filosofica. Roma-Bari: Laterza, 1997. Print.

Svenbro, Jesper. Storia della lettura nella Grecia antica. Roma-Bari: Laterza, 1991. Print. 